P76 (continued)

Conclusions and Implications: Counselors underestimated fruit and vegetable spending, but clients overestimated the adequacy of their fruit and vegetable consumption. Bringing those perceptions in line would likely help the WIC program better serve its clients in Sioux Falls.

Funding: None

\section{P77 Color Me Healthy for SNAP-Ed Supplemental Toolkit: A Comprehensive Approach to the Social-Ecological Model}

Jenelle Wass, MS, LDN, RD, jnwillia@ncsu.edu, North Carolina State University, 516 Brickhaven Drive, Raleigh, NC 27695; Gretchen Hofing, MPH, RD, North Carolina State University; Lindsay Goolsby, MS, LDN, RD;

Lindsey Haynes-Maslow, PhD, MHA;

Carolyn Dunn, PhD, RD

Objective: To enhance SNAP-Ed direct education messages with support for change on the interpersonal and organizational levels of the Social-Ecological Model (SEM).

Theory, Prior Research, Rationale: This project combines the Color Me Healthy for SNAP-Ed direct education program and the Nutrition and Physical Activity SelfAssessment for Child Care (NAP SACC) model, a research-tested intervention, to influence multiple levels of the SEM.

Description: North Carolina Extension educators receive training, on-going support, and an all-inclusive kit for direct education program implementation. Child care centers where Color Me Healthy for SNAP-Ed is delivered are engaged through a comprehensive toolkit for policy, systems, and environmental (PSE) change. This includes self-assessment, guided action planning, staff training, connections with local partners, and the provision of resources. Centers receive hands-on support throughout the process, creating personal connections that build trust and allow for greater center buy-in and a high completion rate, currently $82 \%$. Additionally, one-third of centers have participated for at least two years, fostering a longterm relationship.

Evaluation: The project encourages short, medium, and long term change on the individual and environmental levels, as defined by the SNAP-Ed Evaluation Framework. Administered twice in each annual cycle, NAP SACC, a reliable, valid, environmental self-assessment tool, was used to measure adoption and promotion of nutrition and physical activity supports, while surveys of parents and teachers captured childrens' behavior change.

Conclusions and Implications: The comprehensive application of the toolkit combined with direct education creates a multi-component, multi-level intervention that enhances the effectiveness and sustainability of PSE change at early child care centers.

Funding: USDA

\section{P78 Creating Healthy Behaviors Among Utah's SNAP-Ed Participants}

Casey Coombs, RD, casey.coombs@usu.edu, Utah State University Extension, SNAP-Ed, 8749 Old Main Hill, Logan, UT 84322; Mateja Savoie-Roskos, PhD, MPH, RD, Utah State University; Heidi LeBlanc, MS

Objective: To evaluate the curriculum's effectiveness by determining intention to change behaviors and actual behavior change of SNAP-Ed participants in Utah after receiving nutrition education based on the CREATE Curriculum. A convenience sample of SNAP-Ed participants was surveyed in 2014 and 2015. Participants were surveyed up to three points in time; after participation in one SNAP-Ed class $(n=6,825)$, after receiving at least 6 SNAP-Ed classes $(n=168)$, and 6 -months after receiving at least one SNAP-Ed class $(n=249)$. Participants were asked questions about dietary intake, physical activity, and cooking/mealtime habits. Wilcoxon signed rank test was used to compare nutrition-related behaviors in retrospective pre/post surveys. Participants reported an intention to improve all nutrition-related behaviors in pre-post surveys during the intervention $(\mathrm{p}<0.001)$. Participants also reported actually changing these behaviors six-months post-intervention $(p<0.001)$. After receiving six SNAP-Ed classes participants reported consuming more whole grains, fruits and vegetables and less saturated fats and processed food.

Description: The CREATE Curriculum is an innovative cooking-skills based curriculum developed by Utah's SNAP-Ed program aimed at increasing knowledge, skills, and self-efficacy of participants to help them make healthful nutrition-related decisions to prevent obesity and chronic diseases.

Results: The CREATE Curriculum may be an effective nutrition education tool for improving behaviors associated with a reduced risk of obesity among SNAP-Ed participants. SNAP-Ed programs nationwide should consider utilizing this curriculum in future programming. Future studies should follow SNAP-Ed participants over time to determine if behavior change is sustained.

Funding: Supplemental Nutrition Assistance Program Education

\section{P79 Dairy and Whole Grain Intake Differ by Food Security Status Among Indiana SNAP-Ed Eligible Adults}

Rebecca Rivera, MPH, rcusack@purdue.edu, Purdue University, 700 West State Street, West Lafayette, IN 47907; Melissa Maulding, MS, RD, Purdue University; Angela Abbott, MA, RD, CD; Qi Wang, MS; Heather EicherMiller, PhD

Objective: The Supplemental Nutrition Assistance Program-Education (SNAP-Ed) aims to improve the diet quality of low-income populations. Food insecurity 
P79 (continued)

may be an important factor in participant dietary quality, the evaluation of which may be used to inform nutrition education and other interventions directed to the low-income population. The objective of this study was to determine whether food group intake varies by food security status in a sample of Indiana SNAP-Ed eligible adults.

Study Design, Setting, Participants: SNAP-Ed eligible adults age $\geq 18$ y $(n=261)$ from 31 Indiana counties were recruited to participate in this cross-sectional study from August 2015 to May 2016.

Outcome Measures and Analysis: Baseline household adult food security score was assigned using the 10-item United States Household Food Security Survey Module and classified participants as food secure (0-2) or food insecure (3-10). Baseline Healthy Eating Index-2010 (HEI2010) component scores were assigned using dietary data collected from the National Cancer Institute Automated Self-Administered 24-Hour Recall Tool (2014 version with 1-2 days of recalls per participant). Generalized linear regression models and two-sample t-tests were used to estimate differences in HEI-2010 component scores by food security status.

Results: Food security was significantly associated with increased dairy intake compared to food insecurity $(\beta=$ $1.0 ; \mathrm{p}=0.05$ ), and mean whole grain intake (mean \pm SD) was significantly higher in food secure compared to food insecure Indiana SNAP-Ed eligible adult participants $(1.0 \pm 2.9 ; \mathrm{p}=0.02)$.

Conclusions and Implications: These study results highlight a need to increase dairy and whole grain consumption in the SNAP-Ed eligible population, especially those experiencing food insecurity.

Funding: AgSEEd Crossroads, Purdue Health and Human Sciences Cooperative Extension Nutrition Education Program

\section{P80 Development of a Healthy Weight Management SNAP-Ed Nutrition Education Curriculum: Food Talk: Better U}

Sarah Stotz, MS, $R D, C D E$, University of Georgia;

Laurel Sanville, MS, RD; Rachelle Acitelli Reed, PhD;

Whitni McConnell, MS, RD; Mona F. Habibi, PhD;

Jung Sun Lee, PhD, RD, leejs@uga.edu, University of Georgia, 280 Dawson Hall; Department of Foods and Nutrition, Athens, GA 30602

Objective: To develop a culturally tailored, evidencebased obesity prevention program to address high burden of obesity among SNAP-Ed participants.

Description: The theory was social cognitive theory and expanded health belief model. Food Talk: Better U (FTBU) is a series of four 90-minute sessions taught by paraprofessionals focusing on improving healthy weight management practices. Lesson topics include: portion control, setting SMART goals, limiting added sugar intake, making small healthy shifts in everyday food choices, and problem-solving strategies for mitigating common barriers to healthful eating. Each lesson includes a sharing session, didactic lesson, physical activity (PA), cooking demonstration/tasting, and goal setting. Featured recipes focus on healthful versions of traditional Southern dishes. PA includes resistance exercises with emphasis on proper mechanics. Participants receive a take-home workbook for food and PA journaling, goal setting, additional physical activities, and nutrition education/PA extenders to enhance healthful practices at home (e.g., measuring cups, resistance bands).

Evaluation: A rigorous needs assessment including literature review and interviews with SNAP-Ed eligible participants and peer nutrition educators was completed prior to FTBU development. Formative evaluation was completed in a pilot county, including class observations, participant focus group, and educator interviews. Conclusions and Implications: The development of FTBU is based on systematic, comprehensive needs assessment and rigorous evaluation methods, as aligned with the new direction of SNAP-Ed guidance. FTBU will serve as a foundation for obesity prevention intervention for SNAP-Ed programming in Georgia and will be expanded to eLearning and social marketing nutrition education interventions.

Funding: Supplemental Nutrition Assistance Program Education

\section{P81 EFNEP's Families Eating Smart and Moving More: Integration of Video, Social Media, and PSE in EFNEP Delivery}

Lorelei Jones, MEd, lorelei_jones@ncsu.edu, NC State University, 516 Brickhaven Drive, Raleigh, NC 27695; Carolyn Dunn, $P h D, R D$, NC State University; Emily Foley, MS; Mary Burghardt, MS, LDN, RD, NC Department of Health and Human Services; Lori Rhew, MS, CHES; Suzanne van Rijn, MAEEd, NC State University; Josephine Cialone, MS, RD, NC Department of Health and Human Services; Kathy Andersen, $M S, R D$;

Sheree Vodika, MA, LDN, RD, NC Alliance of YMCAs

Objective: To revise a comprehensive curriculum using current evidence in nutrition, physical activity, behavior change, and adult education that integrates participant and partner engagement strategies to meet the national priorities of EFNEP. Target Audience is EFNEP eligible adult audiences with an emphasis on parents and other adult caregivers who have primary responsibility for feeding children in their care and pregnant/lactating women.

Theory, Prior Research, Rationale: The curriculum is based on Stages of Change and Social Cognitive Theory (SCT) within the framework of the Community Nutrition Education (CNE) Logic Model. The curriculum is designed with a focus on individual, family, or household level change with partner engagement strategies for policy and environmental change informed by the CNE Logic Model.

Continued on page S55 